\title{
A LINEARIZED NUMERICAL SOLUTION FOR STEADY-STATE SIMULATIONS OF GAS NETWORKS
}

\author{
I. Zalitis ${ }^{1 *}$, A. Dolgicers'1 , L. Zemite'1, S. Ganter², \\ V. Kopustinskas ${ }^{3}$, B. Vamanu ${ }^{3}$, I. Bode ${ }^{1}$, J. Kozadajevs ${ }^{1}$ \\ ${ }^{1}$ Riga Technical University, Institute of Power Engineering, \\ 12-1 Azenes Str., Riga, LV-1048, LATVIA \\ ${ }^{2}$ Fraunhofer Institute for High-Speed Dynamics \\ Am Klingelberg 1, Efringen-Kirchen, 79588, GERMANY \\ ${ }^{3}$ European Commission, Joint Research Centre (JRC), Ispra, ITALY \\ *e-mail: Ivars.Zalitis@rtu.Iv
}

Considering the changes of gas transmission system (hereinafter - GTS) brought about by diversification of gas suppliers, new interconnections with European GTS and implementation of an open electricity market and then an open gas market, a steady-state GTS modelling tool has been developed for future implementation in the risk and resilience analysis and potentially operational planning for different GTS or other purposes. The developed method combines the linearized hydraulic conductivity approach with a technique, derived from a linear electrical circuit analysis and an additional pressure change term for modelling of active non-pipeline elements of GTS. This method also takes into consideration operational limits of compressors and pressure regulators and changes in compressibility factor and gas viscosity based on the gas composition, temperature and pressure. The paper includes part of the results obtained from a validation case study performed for the presented method.

Keywords: Modelling, natural gas, steady state, transmission system.

\section{INTRODUCTION}

While the European Union (hereinafter the EU) moves towards its clean energy 2030 and 2050 targets [1]-[3], the EU remains heavily dependent on the natural gas as a primary energy source constituting approximately $22 \%$ of European energy consumption in 2018 [4]. Given its limited and decreasing reserves of natural gas, the $\mathrm{EU}$ is a net importer of gas. The increasing dependency on natural gas imports [4] has posed challenges and increased the risks to security of supply. 
The Baltic GTS is becoming more integrated into the European GTS and it faces similar dependence on gas imports. However, for a long time the Baltic countries had only one major gas supplier (Gazprom) resulting in heavy reliance on this source. This issue is being addressed by building of new interconnections (Estonia-Finland, Lithuania-Poland, Poland-Denmark), [5] LNG terminals (Klaipeda, other potential terminals under consideration) and implementing an open gas market [6].

In Latvia, electricity and heat generation from gas-fired power plants makes up approximately $60 \%$ of the total gas consumption [6]. These existing power plants are now being challenged by low-priced electricity imports after joining the Nord Pool electricity market and increase of interconnectedness of electric grids with the Nordic region. The resulting competition requires more flexibility from the existing gas-fired power plants [7], which automatically affect a large portion of total gas demand in the country. The necessity of flexibility provided by new interconnections between GTS, local gas storage and LNG terminals will only increase after the desynchronisation of the Baltic electric power grid from BRELL [6]. Considering the aforementioned issues, it is clear that the increased complexity of the GTS operation requires new approaches and analysis tools not only for operational planning but also for risk, resilience analysis and reliability assessment.

As a foundation for such an analysis, it is usefull to have a modelling tool allowing for evaluation of the physical operation of GTS, which is often implemented as a single-phase, one-dimensional, isothermal steady-state model (hereinafter - SSM) of a compressible gas flow [8]-[12]. Typically SSM equations are composed using the 1 st or 2nd Kirchof's law and nonlinear flow equations linking GTS element flow rate to its inlet and outlet pressures. Depending on the Kirchof's law used, the Newton-nodal or Newton-loop or Newton loop-node method may be used for formulation and solution of SSM equations [10], [12], [13]. Similar to the Hardy Cross method pre-dating these methods, the Newton-Raphson method variations are known to have poor convergence and strong reliance upon having a good initial guess [8], [9], [11]-[13]. While there are some globaly convergent strategies that may be used to alleviate this issue, the risk of divergence still remains [8]. As can be seen from [12], the main differences of many published SSM versions are the type of flow equation used and GTS aspects (node elavation, possibility to model nonpipeline elements). In this regard, the SSM variant given in [14] is unusual as it combines the Newton-Raphson method with a recalculation of the gas specific gravity to analyse the impact of injection of different gases into the GTS.

Another group of methods applies linearization for their SSM implementation. One method applied for GasGrid software is the Linear Theory method. Here a set of aproximate linearized SSM equations are obtained by changing the difference of pressure squares to pressure drop and average pressure product and flow rate square to flow rate and its previous iteration flow rate product [11]. However, it can also have convergence issues due to numerical oscilations [8]. There is a method, which separates the linear equations obtained using Kirchof's laws and the nonlinear flow equations applying linearisation to the nonlinear part of the SSM [12]. Accurate results are presented with fast convergence but only for particular pipeline networks, but recently another paper has added an approach for modelling of non-pipeline elements [15]. A different method adapts the hydraulic 
conductivity of GTS elements so that the flow rate value remains the same, but it is a function directly of presure drop or drive power in case of compressors [8], [9]. Such an approach is not the easiest one for implementation of compressor constrains, and it can lead to compressor operation outside the permittable operation region. However, this method provided comperatively close results to measurement data from a real pipeline network, which combined with the robust convergence was also integrated in the developed method.

There are also methods for modelling of GTS transients [16], [17], including for a multi-phase flow [18]. While more accurate from the physical prespective, the use of transient simulations for risk and resilience analysis might result in overly large computational burden when considering real-life GTS models that are large and/or detailed.

The main contribution of this paper is the GTS steady-state modelling method that combines the linear hydraulic conductivity analogue approach with derivatives from a linear electrical circuit analysis not only for compilation of SSM equation system, but also for a different, "electrical" approach to modelling of active non-pipeline elements (mainly compressors, pressure regulators). At the same time, the presented method updates gas parameters (compressibility factor, dynamic viscosity) based on gas pressure, temperature and gas composition and takes into consideration operational limitations of pressure regulators and compressors ensuring correct account for transported media and equipment physical parameters. Since the method presented reduces the core task to the solution of system of linear equations, it is highly suitable for modern multicore computing systems. This paper also presents two different flow equations and results from a validation case study using both equations.

\section{THE THEORETICAL BACKGROUND}

Similar to other GTS steady-state modelling methods, the purpose of the developed method is to obtain steady-state node pressures and branch (edge) volumetric flow rates. Considering the challenges of the Latvian GTS described above, this method has to be robust and fast as it will have to operate with a large number of scenarios with varying degrees of difficulty to convergence of the SSM solver. Additionally, this method has to be capable of considering at least some of the main aspects defining the operation of modern GTS:

- complex network topologies;

- potential presence of compressors and pressure regulators;

- existence of several gas suppliers;

- effect of the terrain elevation.
Based on these requirements, it was decided to use a combination of the pipeline hydraulic conductivity linearization approach [8], [9] and an analogy of the mathematical modelling of linear electrical circuits [19], [20].

First, the general form of the equivalent circuit branch used for the developed method to represent elements of GTS will be presented (Fig. 1).

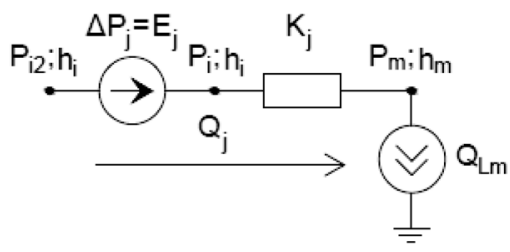

Fig. 1. Model of a GTS element as a branch with a constant volumetric load at the output node. 
Here the typical pipeline model with input pressure $P_{\mathrm{i}}$ and elevation $h_{\mathrm{i}}$, output pressure $P_{\mathrm{m}}$ and elevation $h_{\mathrm{m}}$, hydraulic resistance coefficient considering elevation change $K_{j}$ and volumetric flow rate $Q_{j}$ is extended with an additional pressure change or pressure "source" $\Delta P_{j}=E_{j}$, which can be viewed as an electromotive force source in electrical terms, and constant volumetric flow load $Q_{\mathrm{Lm}}$, which is represented as an electrical current source here. The electrical ground in the figure represents atmospheric pressure, which will serve as a base node for "equivalent circuits" of the GTS. The pressure source $E_{j}$ (that can be either constant or controlled in the loop of the numerical solution process) is added for representation of different non-pipeline elements such as actual network sources with or without their regulators; compressors; pressure regulators etc. The constant flow injections or loads represented with the current source are mainly added for representation of gas consumers and sources, which only provide a fixed amount of gas.

The extended branch model allows creating equivalent circuits for GTS, but in order to calculate the steady-state parameters (node pressures and branch flow rates) a corresponding equation system has to be compiled. As the pipeline steady-state flow equations are nonlinear [10], [21], in order to apply the approach used for linear electrical circuits modified versions of the pipeline hydraulic conductivities corresponding to linear flow equation have to be obtained. Later this transformation will be described in more detail. When these "linearized" conductivities are introduced, one can use the analogy of the Ohm's law and Kirchhoff's first law to compile a linear equation system in the matrix form, in the same manner as the nodal potential or admittance method in electrical circuits is derived [19], [20]:
$Y P=Q_{\mathrm{L}}-M L E+Y_{\mathrm{B}} \mathrm{P}_{\mathrm{B}}$,

$Y=M L M^{\mathrm{T}}$,

where $P$ - the vector of absolute pressures of an equivalent circuit nodes except for the base node, $\mathrm{Pa} ; P_{\mathrm{B}}$ - the absolute pressure of the base node, $\mathrm{Pa} ; Q_{\mathrm{L}}$ - the vector of constant volumetric node demands or injections, $\mathrm{m}^{3} / \mathrm{s} ; Y$ - the matrix of nodal linearized hydraulic conductivities (admittances); $Y_{\mathrm{B}}$ - the base node linearized hydraulic conductivity vector; $M-$ the first-incidence matrix of the network topology graph; $M^{\mathrm{T}}$ - transpose of matrix $M ; L$ - the diagonal matrix of linearized branch conductivities; $E$ - the vector of pressure sources, Pa.

The input matrices for (1) and (2) are composed according to the equivalent circuit of the GTS based on the following rules:

- an element $M_{i j}$ of matrix $M$ assumes value -1 if the flow of branch $j$ flows into the node $i$, value 1 if the current of the branch $j$ flows out of the node $i$ and value 0 if the branch $j$ is not connected to the node $i$;

- an element $Q_{\mathrm{L} i}$ of the vector of $Q_{\mathrm{L}}$ assumes value $Q$ if a flow injection source of $\mathrm{Q} \mathrm{m} / \mathrm{s}$ is connected to and directed towards the node $i$, value $-\mathrm{Q}$ if it is connected to and directed away from the node $i$ (representation of loads) and value $0 \mathrm{~m}^{3} / \mathrm{s}$ in other cases;

- an element $E_{j}$ of the vector $E$ assumes value $E 1$ if an additional pressure increase of $\mathrm{E} 1 \mathrm{~Pa}$ is intended along the assumed flow direction of the branch $j$, value $-\mathrm{E} 1$ if the pressure decrease of $\mathrm{E} 1 \mathrm{~Pa}$ is intended along the branch $j$ and value $0 \mathrm{~Pa}$ in other cases;

- a diagonal element $L_{j j}$ of the matrix $L$ assumes values of corresponding linearized conductivity of the branch $j$, while all non-diagonal elements are zeros; 
- an element $Y_{\mathrm{B} i}$ of the vector $Y_{\mathrm{B}}$ assumes a value of the total linearized conductance of the branches connecting the base node and the node $i$, and value 0 if the node $i$ is not connected to the base node.

In order to obtain the unknown node pressures, one must then solve the linear equation system (2), which can be done in multiple ways [22]. When the node pressures have been obtained, the branch flow rates can be determined using Ohm's law analogy in matrix form:

$Q=L\left(E+M^{T}\left(P-P_{\mathrm{B} 2}\right)\right)$,

where $Q-$ a vector of branch flow rates, $\mathrm{m}^{3} / \mathrm{s} ; P_{\mathrm{B} 2}-$ a vector of the absolute pressure of the base node sized the same as $P, \mathrm{~Pa}$.

Before addressing the linearization of the hydraulic conductivities of the pipelines, at least two often used forms of pipeline flow equations considering elevation described in literature should be presented [9], [10], [21], [23]:

$$
\begin{aligned}
& P_{\mathrm{i}}^{2}-P_{\mathrm{m}}^{2}=K_{1 j}\left(P_{\mathrm{i}}+P_{\mathrm{m}}\right)^{2}+K_{2 j} Q_{j}^{2}, \\
& P_{\mathrm{i}}^{2}-e^{s_{j}} P_{\mathrm{m}}^{2}=K_{3 j} Q_{j}^{2},
\end{aligned}
$$

where $P_{\mathrm{i}}, P_{\mathrm{m}}-$ absolute pressures at the pipeline input and output, $\mathrm{Pa} ; Q_{j}-$ volumetric flow rates of the pipeline, $\mathrm{m}^{3} / \mathrm{s}$; $K_{1 j}$ - flow invariant coefficient of elevation caused pressure change; $K_{2 j}$ - elevation invariant hydraulic resistance coefficient of the pipeline; $K_{3 j}$ - hydraulic resistance coefficient of the pipeline that considers also pipeline elevation; $s_{j}$ - elevation adjustment parameter.

Both (4) and (5) are nonlinear, as mentioned before. Therefore, to apply the analogy with linear electrical circuits, the developed method will adopt the approach of the linearized pipeline hydraulic conductivity analogue, which transforms real pipeline conductivities to ones that result in the same flow rate not due to square root of the difference of pressure squares [8], [9]:

$Q_{j}=C_{j} \sqrt{P_{\mathrm{i}}^{2}-e^{s_{j}} P_{\mathrm{m}}^{2}}, C_{j}=\sqrt{1 / K_{3 j}}$,

but directly due to the pressure drop:

$Q_{j}=L_{j}\left(P_{\mathrm{i}}-P_{\mathrm{m}}\right)$,

where $C_{j}-$ the actual pipeline hydraulic conductivity; $L_{j}$ - the linear analogue of the pipeline hydraulic conductivity.

The value of linear analogue $L_{j}$ is obtained using a transformation coefficient $T_{j}$. When using Eq. (5) or (6) $T_{j}$ is equal to [8], [9]:

$T_{j}=L_{j} / C_{j}=\sqrt{\left(r_{j}^{2}-e^{s_{j}}\right) /\left(r_{j}-1\right)^{2}}$,

where $r_{j}-$ a ratio of the pipeline input and output pressure $\left(r_{j}=P_{\mathrm{i}} / P_{\mathrm{m}}\right)$.

If the term is relocated in the flow equation [24], [25] the transformation coefficient is

$T_{j}=\sqrt{\left(r_{j}^{2} e^{-s_{j}}-1\right) /\left(r_{j}-1\right)^{2}}$.

In case Eq. (4) is used, this coefficient is

$T_{j}=\sqrt{\left(r_{j}^{2}-1-K_{1 j}\left(r_{j}+1\right)^{2}\right) /\left(r_{j}-1\right)^{2}}$,

Equations (8)-(10) show that the function $T_{j}=f\left(r_{j}\right)$ has a break point when $r_{j}=1$ p.u. In order to avoid risk of an infinite $L_{j}$ value, the developed method will change any $1 \leq r_{j}<1.0001$ p.u. to $r_{j}=1.0001$ p.u. Additionally, if $r_{j}<1$ p.u. (actual flow is opposite to the assumed branch direction) $T_{j}$ will be an imaginary number. Thus, in 
such cases the value of pressure ratio $r_{j}$ will inversed to adapt to the actual flow direction. The derived equations (8)-(10) may be used with the GTS element model (Fig. 1), but the pressure ratio must be calculated as follows:

$r_{j}=\left(P_{i 2}+E_{j}\right) / P_{m}$,

where $P_{\mathrm{i} 2}-$ the absolute pressure of the extended GTS element branch model input, $\mathrm{Pa}$.

This modification is especially important for modelling of active or pressure changing non-pipeline elements, for which the conductivity transformation must apply only on the small equivalent pipeline section representing the connecting piping right after this GTS element.

In order to model the pipelines or their sections, two different pipeline flow equations were considered and tested during the development of the proposed method. The first pipeline flow equation form is (4) with coefficients $K_{1 j}$ and $K_{2 j}$ derived from [23]:

$$
\begin{aligned}
& K_{1 j}=g\left(h_{\mathrm{m}}-h_{\mathrm{i}}\right) /\left(2 Z_{\mathrm{avg}} R_{\mathrm{gas}} T_{\mathrm{avg}}\right), \\
& K_{2 j}=\left(\frac{4 P_{\mathrm{st}}}{\pi Z_{\mathrm{st}} T_{\mathrm{st}}}\right)^{2}\left(\frac{f L Z_{\mathrm{avg}} T_{\mathrm{avg}}}{D^{5} R_{\mathrm{gas}} E_{\mathrm{p}}^{2}}\right),
\end{aligned}
$$

where $g$ - gravitational acceleration, $\mathrm{m} / \mathrm{s}^{2}$; $h_{i}, h_{m}$ - elevation of the pipeline beginning and end, $\mathrm{m} ; Z_{\text {avg }}$ - average gas compressibility factor along the pipeline; $T_{\text {avg }}$ - average gas temperature along the pipeline, $\mathrm{K}$; $R_{\text {gas }}$ - specific gas constant, $\mathrm{J} /(\mathrm{kg} \cdot \mathrm{K}) ; L-$ length of the pipeline, $\mathrm{m} ; D$ - inner diameter of the pipeline, $\mathrm{m} ; f$ - the Darcy friction factor; $E_{\mathrm{p}}-$ the hydraulic efficiency of the pipeline, p.u.; $P_{\mathrm{st}}-$ gas pressure at the standard conditions, $\mathrm{Pa} ; T_{\text {st }}$ - gas temperature at the standard conditions, $\mathrm{K} ; Z_{\text {st }}$ - gas compressibility factor at the standard conditions.
The second form considered is (5) with resistance coefficient $K_{3 j}$ [24], [25]:

$$
\begin{aligned}
& K_{3 j}=\frac{4.323 \cdot 10^{-2} f \rho_{\text {st gas } L Z_{\mathrm{avg}} T_{\mathrm{avg}}\left(1-e^{-s_{j}}\right)}}{D^{5} E_{\mathrm{p}}^{2} s_{j}}, \\
& s_{j}=2 g\left(h_{\mathrm{m}}-h_{\mathrm{i}}\right) /\left(Z_{\mathrm{avg}} T_{\mathrm{avg}} R_{\mathrm{gas}}\right),
\end{aligned}
$$

where $\rho_{\text {st }}$ - gas density at the standard conditions, $\mathrm{kg} / \mathrm{m}^{3}$.

The flow equation (5) used with (14), (15) links pressures in $\mathrm{MPa}$ and flows in $\mathrm{m}^{3} / \mathrm{h}$ and for (14) $D$ has to be given in $\mathrm{mm}$. The gas parameters $R_{\text {gas }}$ and $\rho_{\text {st }}$ can be determined either from the specific gravity (relative density) or the molecular weight of the gas [10], [11], [21], [23], [26]-[28]. In order to calculate the compressibility factor, the average pressure along the pipeline based on calculated or initially assumed node pressures is required:

$P_{\mathrm{avg}}=\frac{2}{3}\left(P_{\mathrm{i} 2}+E_{j}+P_{\mathrm{m}}-\frac{\left(P_{\mathrm{i} 2}+E_{j}\right) P_{\mathrm{m}}}{P_{\mathrm{i} 2}+E_{j}+P_{\mathrm{m}}}\right)$,

where $\mathrm{P}_{a v g}$ - the average pressure along the pipeline, $\mathrm{Pa}$.

The compressibility factor and dynamic viscosity are determined according to [25], [26], [29].

The two tested flow equations each use different friction factor estimation approaches. However, both of them require calculation of the Reynolds number [25], [30]:

$R e=10 \rho_{\text {st gas }}\left|Q_{j}\right| /(9 \pi D \mu)$,

where Re - the Reynolds number; $\mu$ dynamic viscosity, $\cdot 10^{-6} \mathrm{~Pa} \cdot \mathrm{s}$.

For a laminar flow $(R e \leq 2000)$ both approaches use the same friction factor formula [21], [23]: 
$f=64 / R e$.

Differences start when the flow is critical, partially turbulent or turbulent. The first approach used in combination with (13) also requires the calculation of the critical Reynolds number [23]:

$R e_{\mathrm{cr}}=35.235\left(\varepsilon_{\mathrm{r}} / D\right)^{-1.1039}$,

where $R e_{\text {cr }}$ - the critical Reynolds number; $\varepsilon_{\mathrm{r}}$ - the equivalent absolute roughness of the pipeline inner wall, $\mathrm{m}$ or $\mathrm{mm}$ (same as $D$ ).

Approximate values of $\varepsilon_{\mathrm{r}}$ for different pipe types are given in [18], [21], [31]. Based on the value of Reynolds number, one of two friction factor formulas is used [23]:

1) if $2000<R e<R e_{\mathrm{cr}}$ the Prandtl-Von Karman equation for partially turbulent is applied:

$1 / \sqrt{f}=-2 \log _{10}(2.825 /(\operatorname{Re} \sqrt{f})) ;$

2) if $R e \geq R e_{\text {cr }}$ the Nikuradse equation for fully turbulent flow is utilised:

$1 / \sqrt{f}=-2 \log _{10}\left(\left(\varepsilon_{\mathrm{r}} / D\right) / 3.7\right)$.

When the flow is partially turbulent, a numerical approach similar to one used for the modified Colebrook-White equation can be utilised to determine the friction factor value [21]. Here this process is repeated until difference $\left(\Delta f=f-f_{0} / f_{0}\right)$ falls below $1 \%$ and the initial guess used is $f_{0}=0.1$. The second approach used with (14) determines the friction factor based both on Reynolds number and the hydraulic roughness of the pipeline inner wall [30], [32]:

1) if $2000<R e \leq 4000$ a formula for critical flow is applied:

$f=0.0025 \sqrt[3]{R e}$
2) if $\operatorname{Re}>4000$ and $\left(\left(\operatorname{Re} \cdot \varepsilon_{\mathrm{r}}\right) / D\right) \geq 23$ a formula for fully turbulent flow in hydraulically rough pipeline is used:

$f=0.11 \sqrt[4]{\left(\varepsilon_{\mathrm{r}} / D\right)+(68 / R e)}$

3) if $4000<\operatorname{Re} \leq 10^{5}$ and $\left(\left(\operatorname{Re} \cdot \varepsilon_{\mathrm{r}}\right) / D\right)<23$ an equation for a partially turbulent flow in a hydraulically smooth pipeline is utilised:

$f=0.3164 / \sqrt[4]{R e}$

4) if $\operatorname{Re}>10^{5}$ and $\left(\left(\operatorname{Re} \cdot \varepsilon_{r}\right) / D\right)<23$ a formula for a fully turbulent flow in a hydraulically smooth pipeline is used:

$f=1 /\left(1.82 \log _{10}(R e)-1.64\right)^{2}$.

In order to model pressure changing non-pipeline elements such as compressors and pressure regulators, the value of the pressure source in the extended GTS element model is determined based on one of the two control strategies. The first one is a constant output pressure strategy, which enforces the pressure at the output (end) node of the branch $\left(P_{\text {out }}=P_{\text {set }}=\right.$ const. $)$, and the second one is a constant compression ratio strategy, which enforces a set value of ratio between the output and input nodes $\left(r_{\mathrm{j}}=P_{\text {out }} / P_{\text {in }}=r_{\text {set }}=\right.$ const. $)$. When the constant output pressure strategy is applied $\left(P_{\text {set }} \neq 0 \mathrm{~Pa}, r_{\text {set }}=1\right.$ p.u. $)$, the pressure source value is calculated as follows:

$E_{j}=\left\{\begin{array}{c}P_{\text {set }}-P_{\mathrm{i} 2}, \text { if } P_{\text {set }}>0 . \\ -\left(\left|P_{\text {set }}\right|-P_{\mathrm{m}}\right), \text { if } P_{\text {set }}<0 .\end{array}\right.$

When the constant pressure ratio strategy is applied ( $r_{\text {set }} \neq 1$ p.u., $\left.P_{\text {set }}=0 \mathrm{~Pa}\right)$, the pressure source value is determined as follows: 
$E_{j}=\left\{\begin{array}{c}E_{j}=P_{\mathrm{i} 2}\left(r_{\mathrm{set}}-1\right), \text { if } r_{\mathrm{set}}>0 . \\ E_{j}=-P_{\mathrm{m}}\left(\left|r_{\mathrm{set}}\right|-1\right), \text { if } r_{\mathrm{set}}<0 .\end{array}\right.$

In case of pipelines and other pasive GTS elements, settings $P_{\text {set }}=0 \mathrm{~Pa}$, $r_{\text {set }}=1$ p.u. are used to indicate that for their branches $E_{j}=0 \mathrm{~Pa}$.

The pressure regulators here are modelled only with the pressure source determined based on a constant output pressure strategy, which directly reflects their actual control mechanism, and a small equivalent pipeline section representing the connecting piping. As most modern pressure regulators are pilot-operated ones with the droop (pressure difference from the setting) in the range of $1-3 \%$ or lower [33], it will be assumed that they are capable to ensure the set pressure at their output. If the inlet pressure of a regulator is to fall below the outlet pressure setting, it is assumed that the regulator will either fully open or go into a bypass mode to retain gas supply to customers as long as possible.

The compressor representation in the equivalent circuits is the same as for pressure regulators. However, for compressors potentially either constant output pressure or pressure ratio strategy may be used, they can have gas self-consumption and their operational limits are more complicated than for pressure regulators. The current version of the developed SSM solver adds the gas self-consumption of a compressor as a percentage of the flow rate through the compressor branch computed in previous iterations. An important aspect for modelling of the compressor steady-state operation is their limits, many of which have been outlined in [10]:

- compression ratio limit: $\left(P_{\text {out }} / P_{\text {in }}\right) \leq r_{\text {max }}$;

- $\quad$ flow rate limit: $Q_{\mathrm{CS}} \leq Q_{\max }$;

- compressor: drive power limit $K W_{\mathrm{CS}} \leq K W_{\mathrm{CSmax}}$;

- $\quad$ out manifold pressure limit $P_{\text {out }} \leq P_{\text {out } \max }$.
After additional literature review, the limit of gas pressure at gas turbine intake $P_{\mathrm{T}} \geq P_{\text {CSmin }}$ was added [34]-[36]. From all the aforementioned limits, the maximum flow rate, outlet pressure and minimum gas pressure for gas turbine driven compressors are implemented as "hard limits" breaching of which will result in compressor shutdown and operation in bypass mode. The other limits are implemented as "soft limits" that essentially change the $r_{\text {set }}$ or $P_{\text {set }}$ values for the corresponding branch. For implementation of the soft limits, different versions of the drive power formula from [21] are used. This compressor representation can also be used for modelling of multi-directional compressor stations either by implementing a valve bridge in the equivalent circuit with one compressor branch or by creating a star connection from branches representing all of the directions of potential pressure increase. In the last case, one must consider that if the limits are exceeded for any of these branches they are breached for the whole compressor station.

The constant $P_{\text {out }}$ strategy is also applied for large balancing sources that can supply large quantities of gas while sustaining pressure at their connection point. At least one of the sources for this method has to be represented with a branch that is connected to the base node and has a corresponding constant output pressure setting.

The developed method has been implemented in an algorithm that has an inner cycle, which first calculates the actual and linearized branch conductivities, then solves (1), obtains branch flow rates using (3), recalculates the hydraulic resistance coefficients with (12)-(15), pressure source values according to (26), (27) and self-consumption of compressors. The inner cycle ends with testing of convergence criteria. In order to exit the inner cycle, one of two criterion groups CRITERION 1 or CRITE- 
RION 2 has to be satisfied. CRITERION 1 requires determination of three parameters:

$$
\begin{aligned}
& E P S_{1}=\max \left(\left|P^{k}-P^{k-1}\right|\right), \\
& E P S_{2}=\max \left(\left|Q_{\text {imb }}\right|\right), \\
& E P S_{3}=\max \left(\left|d_{\text {set }}\right|\right),
\end{aligned}
$$

where $P^{k}, P^{k-1}$ - vectors of absolute node pressures of inner cycle steps $k$ and $k-1, \mathrm{~Pa}$; $Q_{\text {imb }}$ - vector of volumetric node imbalance flows, $\mathrm{m}^{3} / \mathrm{s} ; d_{\text {set }}-$ vector of relative differences between calculated pressures, pressure ratios and corresponding settings $P_{\text {set }}$ and $r_{\text {set }}$, p.u.

Elements of $d_{\text {set }}$ are calculated according to the pressure source control strategy for each branch of an active GTS element:

$$
d_{\text {set } i}=\left\{\begin{array}{c}
\left|\frac{\left(P_{\text {out } i}-P_{\text {set } i}\right)}{P_{\text {set } i}}\right|, \text { if } r_{\text {set } i}=1 \mathrm{p} . \text { u. } \\
\mid \frac{\left(r_{i}-r_{\text {set } i}\right)}{r_{\text {set } i} \mid} \text { if } P_{\text {set } i}=0 \mathrm{~Pa} .
\end{array}\right.
$$

The CRITERION 1 is satisfied if $E P S_{1}$; $E P S_{2} ; E P S_{3}$ are below user defined threshold values $\max E P S_{1}$; $\max E S_{2}$; $\max E P S_{3}$.
CRITERION 2 includes the same test for parameter $E P S_{1}$ but it is compared with a larger threshold $\operatorname{maxEPS}$ to allow for the determination of GTS steady-state parameters even in case of small numerical oscillations that exceed $\max E P S_{1}$. The second parameter used by CRITERION 2 is the number of stagnating steps $N_{\text {stag }}$, which have to exceed a user defined amount of permissible stagnations $\operatorname{maxN}_{\text {stag }}$. The inner cycle step is considered stagnating if neither the $E P S_{1}$ nor $E P S_{3}$ is lower than their minimum values in the previous step and if there is any improvement in either of the two parameters this counter is reset to 0 . If either CRITERION 1 or CRITERION 2 is satisfied within user chosen number of permissible calculation steps $N_{\max }$ the algorithm proceeds to the outer cycle, which checks operational limits of compressors and pressure regulators and if necessary changes the $P_{\text {set }}$ or $r_{\text {set }}$ values accordingly. When there are no compressors and pressure regulators or their parameters are within limits the whole process is finished, but if any of the limits are exceeded the inner cycle will be repeated after the adjustment of $P_{\text {set }}$ or $r_{\text {set }}$ values.

\section{THE VALIDATION CASE STUDY}

A validation case study has been carried out to test the developed method with both flow equations and a method described in [15] and some of the results will be presented here. The settings for the convergence criteria were $\operatorname{maxEPS_{1}}=5 \mathrm{kPA}$ (0.05 bar); $\operatorname{maxEPS}=0.01 \mathrm{~m}_{2}^{3} / \mathrm{s} ; \max$ $E P S_{3}=0.001$ p.u; $\max P S_{4}=20 \mathrm{kPa}$ (0.2 bar). Additionally, the molar fractions of carbon dioxide $x_{\mathrm{CO} 2}$ (with $x_{\mathrm{H} 2 \mathrm{~S}}$ included) and nitrogen $x_{\mathrm{N} 2}$ (with $x_{\mathrm{O} 2}$ included) were assumed to be 0.041 p.u. and 0.04 p.u., respectively, based on composition of natu- ral gas supplied to Latvia [37]-[39].

The first network used for comparison is one given by Leong [8], [9] and includes elevation changes, a compressor and two sources (Fig. 2). Leong assumed that the relative density $S_{\mathrm{g}}=0.69$ p.u.; $E_{\mathrm{p}}=1$ p.u.; $T_{\text {avg }} \approx 297.04 \mathrm{~K} ; T_{\mathrm{st}} \approx 288.71 \mathrm{~K} ; \varepsilon_{\mathrm{r}} \approx 0.046$ $\mathrm{mm} ; Z_{\text {avg }}=0.9$ p.u. for all pipelines in this network. The pipelines represented with flows Q1-Q3 and Q8 are NPS 6 Sch 40 and pipelines represented by branch flows Q4-Q7 and Q9-Q13 are NPS 4 Sch 40. The lengths of the pipelines represented by Q6 
and Q7 are approximately $24.14 \mathrm{~km}$ and for pipelines Q1-Q5, Q8-Q13 approximately $48.28 \mathrm{~km}$ [8], [9].

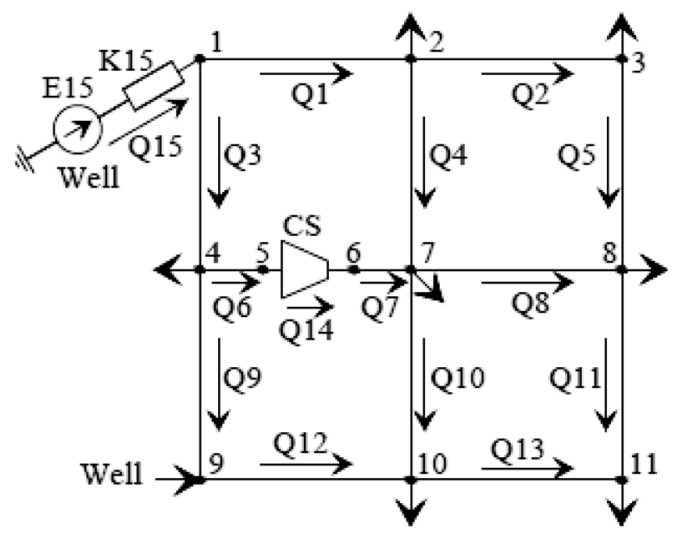

Fig.2. Network diagram for the first example.

For the compressor it was assumed that the inlet side temperature was $297.04 \mathrm{~K}$ and compressibility factor 0.9 p.u. Additionally, the polytrophic coefficient was assumed $n_{\mathrm{p}}=1.4$. The overall compression efficiency was given as $\eta_{\mathrm{p}} \cdot \eta_{\mathrm{CS}}=0.9$ p.u. and the compression ratio setting as $r_{\text {set }}=2.5$ p.u. [8], [9]. For his calculations, Leong used a version of the linearized pipeline hydraulic conductivity method with the AGA-fully turbulent flow equation. The node elevations and gas consumption rates were taken the same as in [8], [9]. An additional branch Q14 was added to model the compressor and the largest of the two gas wells supplying the network was assumed as a balancing source represented with branch Q15 with the output pressure setting the same as pressure calculated by Leong for node 1 (3.511 MPa). The lengths of these artificial pipeline section branches were assumed $1 \mathrm{~m}$ and pipeline diameters were assumed $500 \mathrm{~mm}$.

The node pressure results obtained by the proposed method with flow equation (4) (labelled as "Developed method 1" in graphs) and with modified equation (5) (labelled as "Developed method 2" in graphs), ones obtained by Leong as well as ones calculated using a method developed by Sebastian Ganter [15] are presented in Fig. 3. The calculated flow rates are given below in Fig. 4. When comparing the values of the calculated pressures with ones given by Leong, it was determined that they were within $5 \%$ margin for nodes close to the sources. However, for nodes 3,8 and 11 the obtained pressures differed from Leong's results by $7.6 \% ; 11.3 \%$ and $14.4 \%$ for Ganter's results; $2.3 \%, 3.6 \%$ and $6.6 \%$ for developed method $1 ; 8 \%$, $12.2 \%$ and $17.2 \%$ for developed method 2 . This is largely due to pressure values for these nodes being only $40.0 \% ; 31.2 \%$ and $25.5 \%$ of the source pressure. On the other hand, if the pressure differences were compared to the average network pressure level of $2.236 \mathrm{MPa}$ the deviations of neither the developed method versions nor Ganter's method exceeded $7 \%$. Therefore, one can also see in Fig. 3 that all of the methods obtained practically the same pressure distributions. The multiple correlation coefficient $R^{2}$ values (the closer to 1 the stronger the correlation) calculated according to [40] were $0.9951 ; 0.9992$ and 0.9937 for the Ganter's and developed method 1 and 2, respectively. Similar to Fig. 3, these values also indicate strong correlation between the pressure results. It should be stated that while such a comparison of results is used and close results are a good indicator that the developed methods are on par with already tested ones, an ideal match of results cannot be expected without adjustments of pipeline hydraulic efficiency to account for differences in flow equations and solution methods used for the SSM.

The calculated flow rates for the three methods are mostly within $2 \%$ of ones provided by Leong with the exceptions of Q5 and Q10, which are the two smallest flow 
rates resulting in seemingly large deviations. When these deviations are compared to the average absolute flow rate of the network $\left(1.276 \mathrm{~m}^{3} / \mathrm{s}\right)$, one can see that overall none of the deviations exceeds $2.5 \%$. The overall match between the results can also be seen in Fig. 3 and from the values of the multiple correlation coefficient $\mathrm{R}^{2}(0.9999$, 0.9999 and 1.0000 for the Ganter's and developed method 1 and 2, respectively). The flow rate results show that the difference is significantly smaller, which is to be expected as the flow rates are more predefined than node pressures due to the fixed demand flow rates of the network nodes.

Leong also provided his estimate of the power required by the compressor of 201.81 HP (150.49 kW) [8]. Applying the same assumptions, information on the compressor as used by Leong, the gas compressibility factor values (calculated separately) for the compressor inlet and outlet sides of 0.9609 and 0.9083 , it was possible to calculate the required power as $155.59 \mathrm{~kW}$ according to formula from [21] adopted also by this method. The difference with Leong's result is $3.4 \%$, which indicates that the estimated power necessary for compressor operation is at least similar to one determined with an existing method and could be used for other purposes in the future.

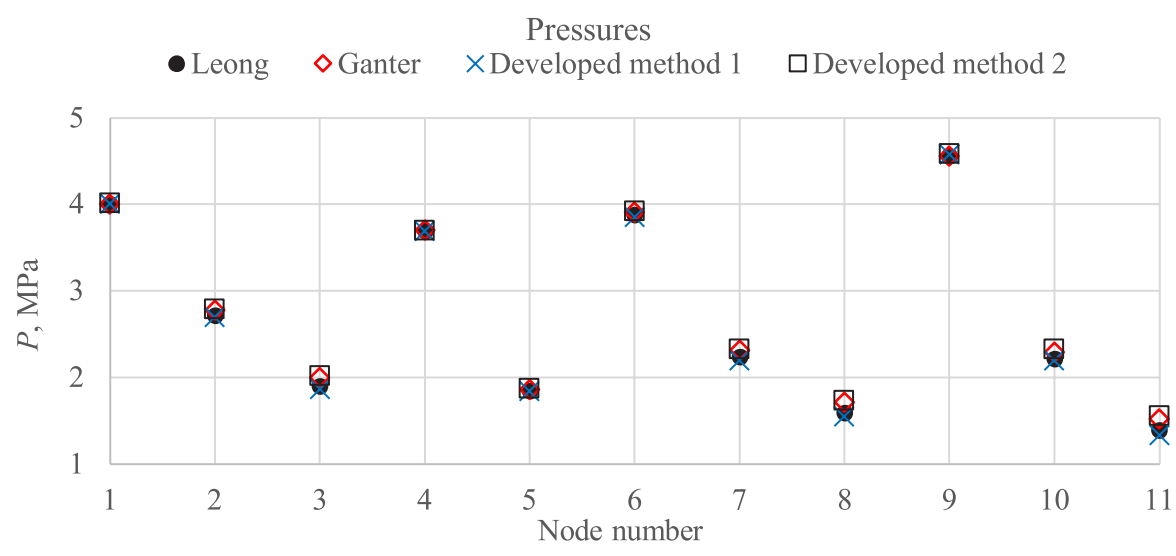

Fig. 3. Graph of the calculated node pressures for the first example.

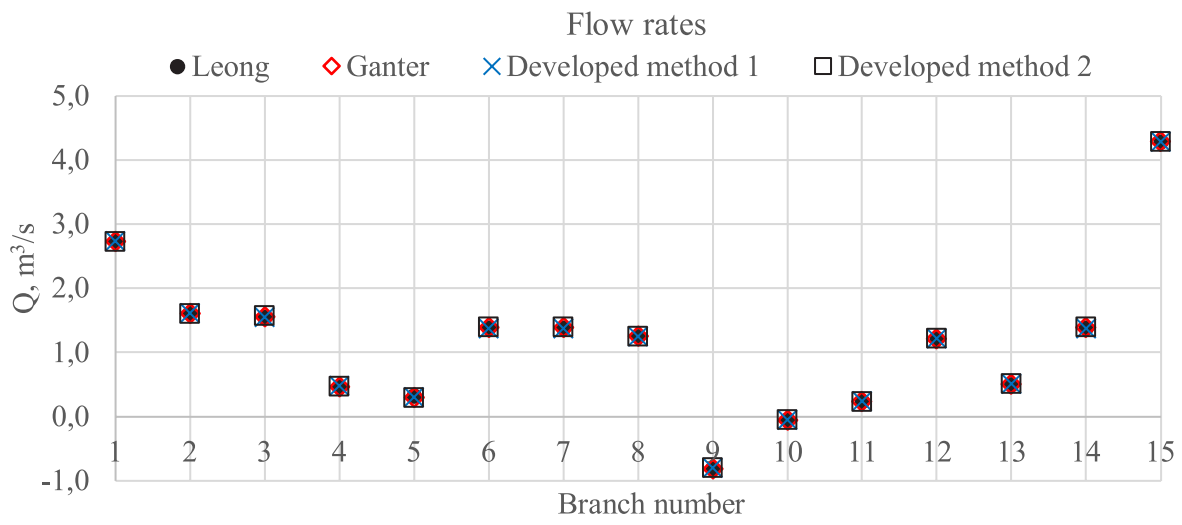

Fig. 4. Graph of the calculated branch flows for the first example. 
The second example that will be presented here is a steady-state simulation of a larger pipeline network (Fig. 5). This network was chosen as it represented a real network in the Mexico valley with the pressure values reported as measurement data [8], [11], [15], [41]. The following assumptions used by the other authors [8], [11], [15], [41] were adopted: $S_{\mathrm{g}}=0.65$ p.u.; $T_{\mathrm{avg}} \approx 191.67 \mathrm{~K}$; $E_{\mathrm{p}}=0.95$ p.u.; $T_{\text {st }}^{\mathrm{g}} \approx 288.71 \mathrm{~K} ; Z_{\text {avg }}^{\text {avg }}=0.98$ p.u. for all of the pipelines in this network. The pipeline lengths and diameters were taken from [8], [9] and the values of pipeline inner wall roughness $\varepsilon_{\mathrm{r}}$ were obtained from [11]. The demand flow rates were taken from Leong's work [8], [9] as ones given in [11] seemed too miniscule to result in the reported pressure drops. The assumptions about the gas composition remain the same as in the first example. The node elevations were unknown or disregarded by the other authors so they were not considered here as well.

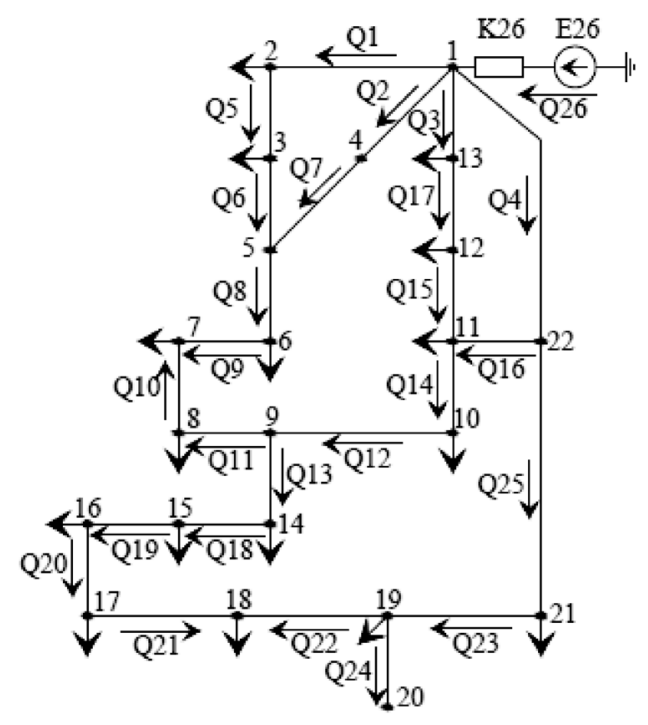

Fig.5. Network diagram for the second example.

The pressure results by Martinez were obtained using the Panhandle-B flow equation and the Newton-Raphson method. He also applied the conjugated gradient method to improve the convergence of the solution of a linear equation system that provided the node pressure correction vector for the Newton-Raphson method [41]. In order to apply the proposed method, a branch represented with the flow Q26 was added to serve as a pressure source with $P_{\text {set }}$ the same as reported node 1 pressure of $2.461 \mathrm{MPa}$. The length of this artificial pipeline section branch was assumed $1 \mathrm{~m}$ and pipeline diameter was assumed $1000 \mathrm{~mm}$.

The node pressures including the reported measurement values [8], [9], ones calculated by Martinez [41] as well as ones obtained using the two versions of the developed method and Ganter's method are given in Fig. 6. The calculated branch flow rate results for comparison were available only from a graph given by Leong [8], [9]. Thus, in Fig. 7 the results of the two versions of the developed method and Ganter's method were compared only to Leong's results. Considering the assumptions made and potential measurement errors, all four modelling methods obtained pressures close to measurement data (largest deviations being $4.41 \%, 5.22 \%, 4.97 \%$ and $5.18 \%$ for Martinez's method, Ganter's method, developed method 1 and developed method 2 , respectively). Figure 6 also shows how well all of the methods captured the significant pressure drop for nodes 15-20 caused by choke points (small diameter pipelines Q18 and Q23). The almost constant pressure value difference for nodes 2-8 seem to indicate that the hydraulic efficiency of pipelines represented with Q1 and Q2 were lower than reported or assumed by other authors. The multiple correlation coefficient $\mathrm{R}^{2}$ values (determined using the measurements as reference) for this example were 0.9987 ; $0.9980 ; 0.9986$ and 0.9981 for the Martinez's method; Ganter's method and developed method 1 and 2, respectively, which also indicated that all of the meth- 
ods replicated the pressure distribution well. One can also notice that the measured pressures were lower than those obtained by modelling methods (including Leong's method [8], which had similar results to ones presented by Martinez). To some extent, this is an expected result as it shows than in fact the real pressure drops are slightly higher, but the difference can and is intended to be accounted for with small changes in the hydraulic efficiency values as described in theory [10], [21].

The calculated flow rates in most cases are within $6 \%$ of ones obtained by Leong [8], [9] with exceptions of Q5; Q6; Q10; Q11; Q21, the value of which are $35 \%$ or less of the average absolute flow rate of the network of $14.659 \mathrm{~m}^{3} / \mathrm{s}$. When compared to this average flow rate, neither of the deviations of branch flow results reach $6 \%$. The overall match of estimated flow rates to ones reported by Leong is also indicated by Fig. 7 and values of the multiple correlation coefficient $\mathrm{R}^{2}(0.9995,0.9997$ and 0.9995 for the Ganter's method and developed method 1 and 2, respectively).

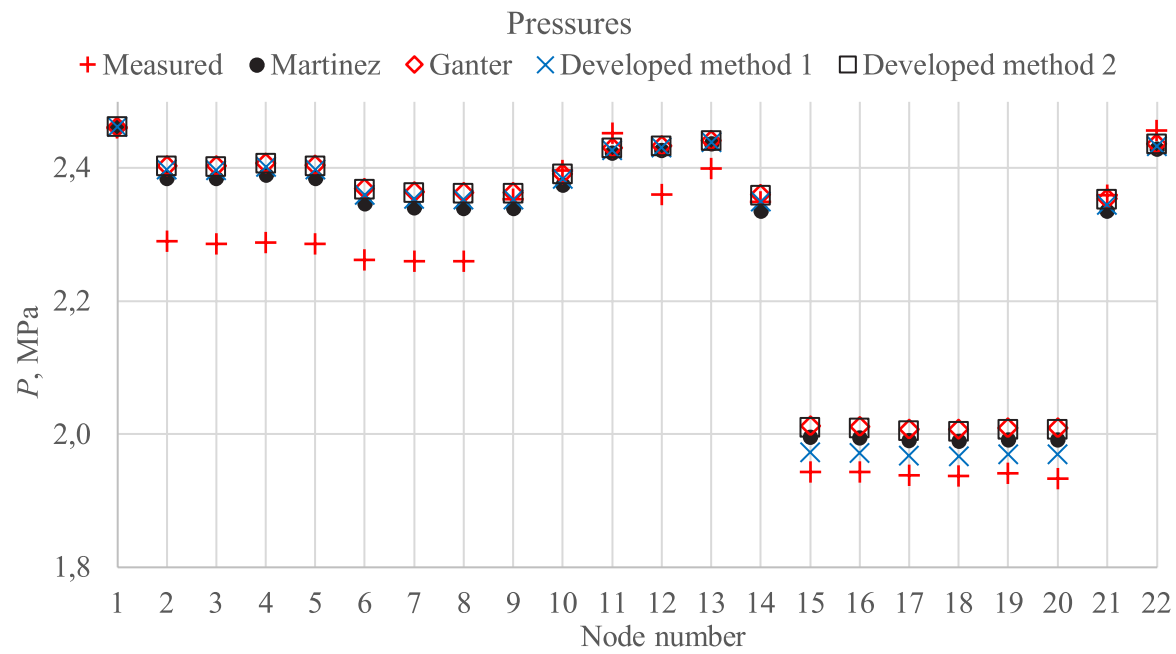

Fig. 6. Graph of the measured and calculated node pressures for the second example.

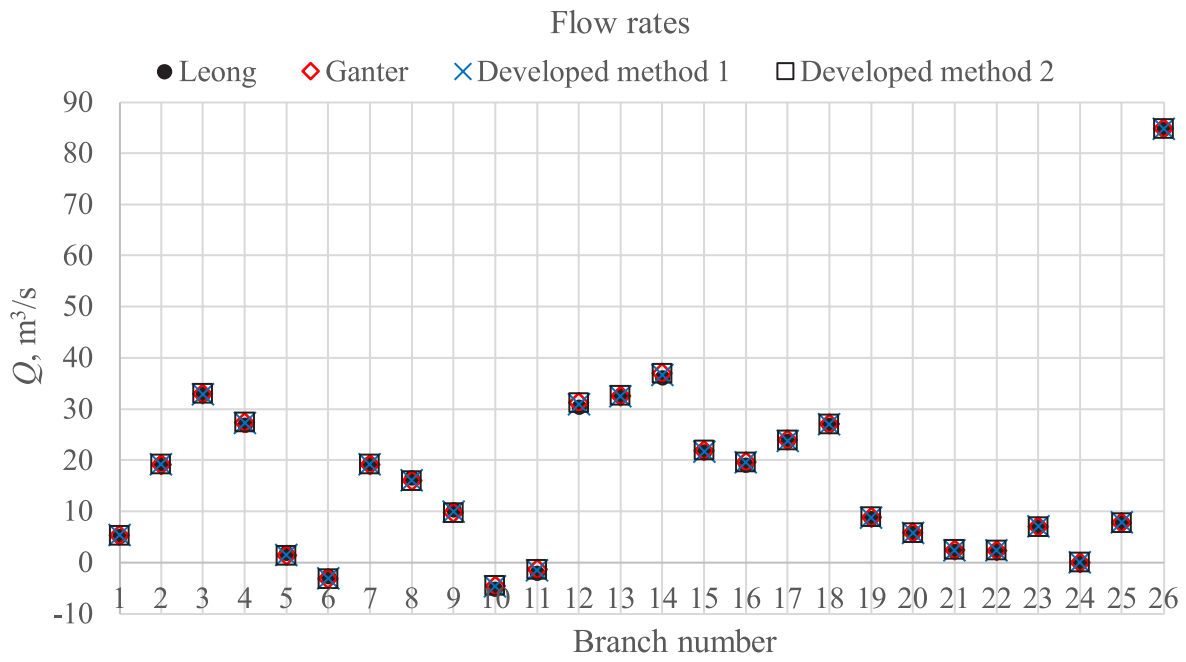

Fig. 7. Graph of the calculated branch flow rates for the second example. 
1. The combination of analogy of graph based steady-state modelling of linear electrical circuits with linearized hydraulic conductivity analogue can be implemented in GTS SSM solver that is straightforward in terms of use and robust due to the linear nature of the main equation system.

2. Use of an additional pressure change term in the steady-state flow equation and determination of its value according to either constant output pressure or constant pressure ratio control strategy provides a flexible and easy-to-use approach for modelling of the main active non-pipeline elements of GTS (compressors, pressure regulators, etc.).

3. The applied pressure change control strategies with their settings can be utilised to implement operational limits of pressure regulators and compressors both by adjusting these settings and representing the bypass mode.

4. The validation case study showed that the flow rates estimated with the developed method and Ganter's method in most cases were well within $5 \%$ of the reported ones or at least the average flow rate value and their general match to reported results were further confirmed by values of the multiple correlation coefficient.
5. The obtained node pressure values in most cases were within $6 \%$ of the ones reported for validation study examples except the cases where the reported pressure value was already relatively small, but the differences were always within $7 \%$ of the average pressure level of the network nodes.

6. Due to diverse flow equations and mathematical solution methods used for the SSM as well as potential deviations between assumptions made and the actual network parameters, some differences between the obtained and previously reported results were to be expected and, therefore, the main goal during this validation process was to obtain a similar flow rate and pressure distributions with limited (say up to 5-10\%) differences that could be accounted for by small changes in the hydraulic efficiency of the pipelines.

7. Considering the aforementioned aspects and the relatively good match between the obtained results and the results reported for both a theoretical and a real GTS network case, it can be concluded that both the developed method and Ganter's method were validated.

\section{ACKNOWLEDGEMENTS}

This research has received funding from the European Union's Horizon 2020 research and innovation programme under grant agreement No. 833017 (project "SecureGas").

The research has also been supported by the National Research Programme, project "Trends, Challenges and
Solutions of Latvian Gas Infrastructure Development (LAGAS)" (No. VPP-EMINFRA-2018/1-0003), and by the Ministry of Economics of the Republic of Latvia, project "Innovative Smart Grid Technologies and their Optimization (INGRIDO)" (No. VPP-EM-INFRA-2018/1-0006). 


\section{REFERENCES}

1. European Commission. (2019). Communication from the Commission to the European Parliament, the Council, the European Economic and Social Committee and the Committee of the Regions: United in Delivering the Energy Union and Climate Action - Setting the Foundations for a Successful Clean Energy Transition. Brussels: European Commission. Available at https://op.europa.eu/en/publication-detail/-/ publication/62ee9843-92aa-11e9-936901aa75ed71a1/language-en

2. European Commission. (2019). Communication from the Commission to the European Parliament, the Council, the European Economic and Social Committee and the Committee of the Regions: The European Green Deal. Brussels: European Commission. Available at https://ec.europa.eu/info/strategy/ priorities-2019-2024/european-green-deal_en

3. United Nations. (2015). Resolution A/70/1: Transforming our World: the 2030 Agenda for Sustainable Development. United Nations. Available at https://sdgs.un.org/2030agenda

4. European Union. (2020). Energy StatisticsAn Overview. Available at https://ec.europa. eu/eurostat/statistics-explained/index.php/ Energy_statistics_-_an_overview

5. ENTSOG aisbl. (2013). Baltic Energy Market Interconnection Plan GRIP 20142023. Brussels: ENTSOG. Available at https://www.entsog.eu/sites/default/files/ files-old-website/publications/GRIPs/2014/ GRIP_002_140514_BEMIP_2014-2023 main_low.pdf

6. Conexus Baltic Grid. (2020). Natural Gas Transmission System Operator Annual Assessment Report 2019. Riga: Conexus Baltic Grid. Available at https://www. conexus.lv/tso-annual-statement/psoikgadeja-novertejuma-zinojums-par2019gadu

7. Ivanova, P. (2018). Improvement of Flexibility and Efficiency of Thermal Power Plants under Variable Operation Conditions. Doctoral Thesis. Riga: RTU Press. Available at https:// ortus.rtu.lv/science/en/publications/27937
8. Leong, C. Y. (2012). A Robust LinearPressure Analog for the Analysis of Natural Gas Transportation Networks. M.Sc. Thesis, Dept. Energy and Mineral Engineering, The Pennsylvania State University, University Park. Available at https://etda.libraries.psu. edu/catalog/14552

9. Ayala, L. F., \& Leong, C. Y. (2013). A Robust Linear-Pressure Analog for the Analysis of Natural Gas Transportation Networks. Journal of Natural Gas Science and Engineering, 14, 174-184. Available at https://doi.org/10.1016/j.jngse.2013.06.008

10. Osiadacz, A. J. (1987). Simulation and analysis of gas networks. London: E. \& F.N. SPON, 1987.

11. Nagoo, A. S. (2003). Analysis of Steady and Quasi-Steady Gas Flows in Complex Pipe Network Topology. M.Sc. Thesis, Dept. Energy and Geo-Environmental Engineering, The Pennsylvania State University, University Park. doi: 10.13140/2.1.3910.9924

12. Ekhtiari, A., Dassios, I., Liu, M., \& Syron, E. (2019). A Novel Approach to Model a Gas Network. MDPI Applied Sciences, 9 (6), 1-26. Available at https://doi.org/10.3390/ app9061047

13. Shahin, I. A. S. (2012). Optimization of Gas Pipeline Networks Using Genetic Algorithms. M.Sc. Thesis, Dept. Mechanical Power Engineering, The Mansoura University, Mansoura. Available at https://www. researchgate.net/profile/Berge_Djebedjian/ publication/270897194_OPTIMIZATION OF_GAS_PIPELINE_NETWORKS USING_GENETIC_ALGORITHMS in_Mechanical_Power_Engineering_ Supervisors _ Prof_ Berge Ohanness_Djebedjian_and_Dr_ Mohamed__A h med_E 1 naggar / links/54b8e75b0cf28faced625b5f.pdf

14. Abeysekera, M., Wu, J., Jenkins, N., \& Rees, M. (2015). Steady State Analysis of Gas Networks with Distributed Injection of Alternative Gas. Applied Energy, 164, 9911002. Available at https://doi.org/10.1016/j. apenergy.2015.05.099 
15. Ganter, S., Srivastava, K., Vogelbacher, G., Finger, J., Vamanu, B., Kopustinskas, V., ... \& Stolz, A. (2020). Towards risk and resilience quantification of gas networks based on numerical simulation. In Proceedings of the 30th European Safety and Reliability Conference and the 15th Probabilistic Safety Assessment and Management Conference (ESREL2020 PSAM15), (pp.819-826), 1-5 November 2020, Venice, Italy. Singapore: Research Publishing. Available at https://www. rpsonline.com.sg/proceedings/esrel2020/ pdf/3971.pdf

16. Herrán-González, A., De La Cruz, J. M., De Andrés-Toro, B., \& Risco-Martín, J. L. (2009). Modeling and Simulation of a Gas Distribution Pipeline Network. Applied Mathematical Modelling, 33 (3), 15841600. Available at https://doi.org/10.1016/j. apm.2008.02.012

17. Bao, Z., Jiang, Z., \& Wu, L. (2020). Evaluation of Bi-Directional Cascading Failure Propagation in Integrated Electricity-Natural Gas System. Electric Power and Energy Systems, 121, 1-10. Available at https://doi. org/10.1016/j.ijepes.2020.106045

18. Bratland, O. (2009). Pipe Flow 1: Single-phase flow assurance. Bangkok: Self-published. Available at http://www.drbratland.com/

19. Bessonov, L. (1996). Theoretical foundation of electrical engineering. Electrical circuits (9th ed.). Moscow: High School (in Russian).

20. Agarwal, A,. \& Lang, J. H. (2005). Foundations of analog and electronic circuits. San Francisco: Morgan Kaufmann Publishers.

21. Menon, E. S. (2005). Gas pipeline hydraulic. London: CRC Press Taylor \& Francis Group.

22. Chapra, S. C., \& Canale, R. P. (2010). Numerical methods for engineers (6th ed.). London: McGraw-Hill Higher Education.

23. Coelho, P. M., \& Pinho, C. (2007). Considerations about Equations for Steady State Flow in Natural Gas Pipelines. Journal of the Brazilian Society of Mechanical Sciences and Engineering, 29 (3), 262-273. doi: 10.1590/S1678-58782007000300005
24. Gazprom. (2012). Gas Distribution Systems. Standardized Technical Solutions for Creation of Hydraulic Models of Gas Supply Systems. Inner recommendations of JSC "Gazprom" 2-1.17-584-2011 (in Russian).

25. Gazprom. (2013). Determination of Technical and Available Capacity of Gas Pipelines. Inner standard of JSC "Gazprom gas distribution" 12.2.2-1-2013 (in Russian). Available at https://www.politerm.com/ media/zulugaz/news/STO $\% 20$ GP $\% 20$ GR\%2012.2.2-1-2013.pdf

26. Natural Gas. Methods of Calculation of Physical Properties. Definition of Physical Properties of Natural Gas, its Components and Processing Products. GOST30319.196. (1997). (in Russian). Available at http://dtp.lg.ua/Dokumentacija\%20i\%20 oformlenie/GOST-30319.1-96-Gaz\%20 prirodnyiy.-Metodyi-rascheta-fizicheskihsvoystv.-Opredelenie-fizicheskih-svoystvprirodnogo-gaza-ego-komponentov-iproduktov-ego-pererabotki.pdf

27. Engineering ToolBox. Universal and Individual Gas Constants. Available at https://www.engineeringtoolbox.com/ individual-universal-gas-constant-d_588. html

28. ISO. (2016). Natural Gas - Calculation of Calorific Values, Density, Relative Density and Wobbe Indices from Composition. ISO 6976:2016.

29. State System for Ensuring Uniformity of Measurements. Natural Gas. The Coefficient of Dynamic Viscosity of Compressed Gas with a Known Component Composition. The Method of Calculation. National standard of Russian federation GOST R 8.770-2011. (2013). (in Russian). Available at http://www.gostrf.com/normadata/1/ 4293791/4293791887.pdf

30. Design Of External Natural Gas Pipeline Systems with Pressure 1.6 MPA (16 Bar), LVS 417:2015. (2015). (in Latvian).

31. CRANE CO. (1982). Flow of fluids through valves, fittings and pipe metric edition - SI units. Technical Paper No. 410M, (4th ed.). New York: CRANE CO. 
32. Volkov, V. S., Volnov, Yu. N., Gabelya, R. D., Golik, V. G., .... \& Guseva N. B. (2006). The general provisions for design and construction of gas distribution systems using steel and polyethylene pipes. Moscow: Polimergaz (in Russian). Available at https://files.stroyinf.ru/ Data2/1/4294816/4294816188.pdf

33. Emerson Process Management Regulator Technologies Inc. (2015). Natural gas technologies. Application guide - Edition VII. Singapore: Tien Wah Press. Available at https://www3.emersonprocess.com/ Regulators/Natural\%20Gas\%20E-Book/ NG_AppGuide_ebook_output/web/html5/ index.html? \&locale=ENG\&pn=1

34. Strahovich, K. I., Frenke,l M. I., Kondryakov, I. K., \& Ris, V. F. (1961). Compressor machines. Moscow: State Publishing House of Trade Literature.

35. Yevdokimov, V. Ye., Korosov, Yu. G., Stolyarov, A. A., Fomin, V. G., Arhipov, V. V., .. \& Kamenev V. M. (1982). Centrifugal compressor machines and turbines which drives them. Industrial catalogue 12-82. Moscow: Research Institute of Economics, Organisation of Production, Technical and Economic Information of MachineBuilding in Energetics (in Russian).

36. Kozachenko, A. N. (1999). Operation of compressor stations of magistral gas pipelines. Moscow: Oil and Gas (in Russian).

37. Zemite, L., Kutjuns, A., Bode, I., Kunickis, M., \& Zeltins, N. (2018). Consistency Analysis and Data Consultation of Gas System of Gas-Electricity Network of Latvia. Latvian Journal of Physics and Technical Sciences, 55 (1), 22-34. doi: 10.2478/lpts-2018-0003.
38. Zemite, L., Kutjuns, A., Bode, I., Kunickis, M., \& Zeltins, N. (2018). Risk Treatment and System Recovery Analysis of Gas System of Gas and Electricity Network of Latvia. Latvian Journal of Physics and Technical Sciences, 55 (5), 3-14. doi: 10.2478/lpts-2018-0031.

39. Savickis, J., Zeltins, N., \& Jansons, L. (2019). Synergy between the Natural Gas and RES in Enhancement of Security of Energy Supply in the Baltic Countries (Problem Statement). Latvian Journal of Physics and Technical Sciences, 56 (6), $17-$ 31. doi: 10.2478/lpts-2019-0032.

40. Carroll, J. D., Green, P. E., \& Chaturvedi, A. (1997). Mathematical tools for applied multivariate analysis (rev. ed.). San Diego: Academic Press. Available at https://doi. org/10.1016/B978-0-12-160954-2.X5000-8

41. Martinez-Romero, N., Osorio-Peralta, O., \& Santamaria-Vite, I. (2002). Natural gas network optimization and sensibility analysis. In Proceedings: SPE International Petroleum Conference and Exhibition in Mexico, (pp. 1-14), 10-12 February 2002, Vilahermosa, Mexico. Richardson: Society of Petroleum Engineers. Available at https:// doi.org/10.2118/74384-MS 